\title{
Identification of apple cultivars on the basis of simple sequence repeat markers
}

\author{
G.S. Liu ${ }^{1,2}$, Y.G. Zhang ${ }^{2}$, R. Tao ${ }^{3}$, J.G. Fang ${ }^{3}$ and H.Y. Dai ${ }^{2}$ \\ ${ }^{1}$ College of Horticulture \& Landscape, Hunan Agricultural University, \\ Changsha, Hunan, China \\ ${ }^{2}$ College of Horticulture, Qingdao Agricultural University, \\ Qingdao, Shandong, China \\ ${ }^{3}$ College of Horticulture, Nanjing Agricultural University, \\ Nanjing, Jiangsu, China \\ Corresponding author: H.Y. Dai \\ E-mail: hydai@qau.edu.cn
}

Genet. Mol. Res. 13 (3): 7377-7387 (2014)

Received April 8, 2013

Accepted December 5, 2013

Published September 12, 2014

DOI http://dx.doi.org/10.4238/2014.September.12.3

\begin{abstract}
DNA markers are useful tools that play an important role in plant cultivar identification. They are usually based on polymerase chain reaction (PCR) and include simple sequence repeats (SSRs), inter-simple sequence repeats, and random amplified polymorphic DNA. However, DNA markers were not used effectively in the complete identification of plant cultivars because of the lack of known DNA fingerprints. Recently, a novel approach called the cultivar identification diagram (CID) strategy was developed to facilitate the use of DNA markers for separate plant individuals. The CID was designed whereby a polymorphic maker was generated from each PCR that directly allowed for cultivar sample separation at each step. Therefore, it could be used to identify cultivars and varieties easily with fewer primers. In this study, 60 apple cultivars, including a few main cultivars in fields and varieties from descendants (Fuji x Telamon) were examined. Of the 20 pairs of SSR primers screened, 8 pairs gave reproducible, polymorphic DNA amplification patterns. The banding
\end{abstract}


patterns obtained from these 8 primers were used to construct a CID map. Each cultivar or variety in this study was distinguished from the others completely, indicating that this method can be used for efficient cultivar identification. The result contributed to studies on germplasm resources and the seedling industry in fruit trees.

Key words: Apple; Cultivar and varieties; Simple sequence repeat; DNA marker; Cultivar identification diagram

\section{INTRODUCTION}

Apple (Malus x domestica Borkh.) is the most produced temperate tree crop, one of the most widely cultivated fruit trees, and the fourth most economically important fruit tree following citrus, grape, and banana (Hummer and Janick, 2009). There are more than 6000 documented cultivars of apples in the world, but a few major cultivars now dominate the world fruit production (Janick et al., 1996). The genetic base of the cultivated apple has greatly eroded over time as regional cultivars have been replaced. This has been compounded by the loss of many public apple breeding projects and their associated apple cultivar collections (Brooks and Vest, 1985). Most breeders usually hope to gain at least 200-300 seeds per cross, then they seed them and screen seedlings by graft. However, it is difficult to avoid cross contamination from the incomplete emasculation step and pollens from other parents. Cultivar identification and characterization are the first steps of any fruit tree introduction and improvement of seedling screening programs. Therefore, how to use an efficient and workable way to identify cultivars become important for apple cultivar introduction and early identification of crossbred offspring.

In the last few decades, the identification of fruit trees, including apple, was based on morphological, physiological, and agronomic traits. However, traditional cultivar identification based on morphological traits requires extensive observations of mature plants, and, in many situations, it lacks definition and objectivity (Wrigley et al., 1987). Furthermore, morphological traits cannot serve as unambiguous markers because of environmental influences. With the rapid development of molecular markers in the last 2 decades, it solved this difficult problem successfully. On the other hand, unlike biochemical markers with limited polymorphism and spatial-temporal variations, cultivar-specific genetic markers are desirable for cultivar identification for their unique advantage of not being influenced by the physiology or environment.

The choice of the marker system will depend on the type of genomic information required and their ability to detect polymorphisms in a given population. In the past few years, a variety of molecular markers were developed and used for study on genetic diversity, fingerprinting and cultivar origins (Melgarejo et al., 2009; Cheng and Huang, 2009; Elidemir and Uzun, 2009; Papp et al., 2010). Although, restriction fragment length polymorphisms have been used to identify apple clones and seedlings (Nybom and Schaal, 1990; Watillon et al., 1991), it is laborious and not suited for studies of a large number of samples (Williams et al., 1990). Randomly amplified polymorphic DNA markers (RAPD), amplified fragment length polymorphism (AFLP), and microsatellite or simple sequence repeat (SSR) reduced the cost of identifying genetic markers and allowed large scale genotyping of individuals (Ferreira and Grattapaglia, 1995). Among the different types of molecular markers, microsatellite or SSR markers are thought as highly prized molecular markers owing to their co-dominance and high levels of polymorphism (Papa et al., 
2005; Varshney et al., 2005). With the development of DNA markers, more microsatellite or SSR markers have been widely used in major crops (Smith et al., 1997; Röder et al., 1998; Ramsay et al., 2000; Temnykh et al., 2001; Wang et al., 2011) because of their ease of analysis (Powell et al., 1996; Donini et al., 1998). SSR markers may have advantages over those from traditional enriched genomic libraries. Greater transferability of SSR markers between species may be possible because of higher conservation of SSR flanking sequences. The mapping of SSRs will also provide a location, in many cases, for genes of known function. The sequence polymorphisms and mapping of the polymorphic markers identified may correspond directly to genes controlling agronomic traits of interest. Therefore, successful screening of primers for polymorphic SSR markers is the prerequisite for cultivar identification.

In the investigation presented here, we introduce a more workable method based on SSR markers that can make the identification of apple cultivars a practical, efficient, recordable, and easily referable work. A cultivar identification diagram (CID) generated from the SSR banding patterns was constructed manually. It could be used as a source of reference information for apple cultivar identification in the future. Furthermore, the reliability of the SSR-PCR system as a tool to identify apple cultivars was tested.

\section{MATERIAL AND METHODS}

\section{Plant materials and DNA extraction}

A total of 60 varieties were used in this study, including August Fuji, Dounan, Fuyan, Fuji, Guifei, Golden Delicious, and Hongqian Fuji. The names of these cultivars are as listed in Table 1. Young leaves of the 60 apple cultivars selected were collected from Qingdao Agricultural University, Shandong, China. These samples were quickly frozen in liquid nitrogen and stored at $-80^{\circ} \mathrm{C}$. Total genomic DNA of each genotype was extracted from young leaves using the modified cetyltrimethylammonium bromide method (Murray and Thompson, 1980; Bousquet et al., 1990). The DNA extracted was diluted to a final concentration of $30 \mathrm{ng} / \mu \mathrm{L}$ with $1 \mathrm{X}$ Tris-ethylenediaminetetraacetic acid buffer and stored at $-20^{\circ} \mathrm{C}$ until use, and $0.8 \%$ agarose gels were used to check the quality of the DNA.

\section{Primer design}

The whole sequences of the apple genome were screened for SSR motifs using the MISA software. The following screening SSR loci standards were used: dinucleotide, trinucleotide, tetranucleotide, pentanucleotide, and the hexanucleotide smallest repeat numbers were $8,6,4$, and 3. Thirty pairs of apple SSR primers were designed by scanning the SSR location information using the Primer 5.0 plus software. The following main parameters were applied: the $\mathrm{GC}$ content of the primers is 40 to $70 \%$, and the optimum value is $50 \%$; the primer length is 18-24 bp; the optimum annealing temperature is 50 to $65^{\circ} \mathrm{C}$, and the temperature difference of the left and right primers is less than $5^{\circ} \mathrm{C}$; and the product fragment size is $150-350 \mathrm{bp}$. It is essential to evaluate the primers using the Oligo5.0 software and avoid primer dimers, hairpin structures, and mismatches. Twenty SSR-PCR primer pairs were synthesized by the company Generay Biotechnology in Shanghai, China, and were tested with a few genotypes initially. Only those primers resulting in clear unambiguous banding patterns with all genotypes tested were selected for use in genotyping. 
Table 1. Names and origin of the cultivars and varieties used.

\begin{tabular}{|c|c|c|c|c|c|}
\hline Code & Name & Origin & Code & Name & Origin \\
\hline 1 & August Fuji & China & 31 & $95-100$ & China \\
\hline 2 & Dounan & Japan & 32 & $95-103$ & China \\
\hline 3 & Fuyan & China & 33 & $95-109$ & China \\
\hline 4 & Fuji & Japan & 34 & 95-011 & China \\
\hline 5 & Guifei & China & 35 & $95-117$ & China \\
\hline 6 & Hongqian Fuji & Japan & 36 & $95-125$ & China \\
\hline 7 & Hongxun No. 1 & China & 37 & $95-149$ & China \\
\hline 8 & Lujia No. 1 & China & 38 & $95-156$ & China \\
\hline 9 & Lujia No. 2 & China & 39 & $95-016$ & China \\
\hline 10 & Lujia No. 3 & China & 40 & $95-161$ & China \\
\hline 11 & Lujia No. 4 & China & 41 & 95-019 & China \\
\hline 12 & Lujia No. 5 & China & 42 & $95-209$ & China \\
\hline 13 & Lujia No. 6 & China & 43 & $95-210$ & China \\
\hline 14 & Trajan & England & 44 & $95-023$ & China \\
\hline 15 & Telamon & England & 45 & $95-024$ & China \\
\hline 16 & Tuscon & England & 46 & $95-025$ & China \\
\hline 17 & 7C-001 & China & 47 & $95-030$ & China \\
\hline 18 & 7C-100 & China & 48 & $95-032$ & China \\
\hline 19 & 7C-102 & China & 49 & $95-033$ & China \\
\hline 20 & 7C-103 & China & 50 & $95-034$ & China \\
\hline 21 & 7C-104 & China & 51 & $95-036$ & China \\
\hline 22 & $7 C-106$ & China & 52 & $95-041$ & China \\
\hline 23 & $7 C-107$ & China & 53 & $95-045$ & China \\
\hline 24 & $7 C-110$ & China & 54 & $95-054$ & China \\
\hline 25 & 7C-015 & China & 55 & $95-072$ & China \\
\hline 26 & $7 C-150$ & China & 56 & $95-076$ & China \\
\hline 27 & 7C-035 & China & 57 & $95-078$ & China \\
\hline 28 & $7 C-043$ & China & 58 & $95-085$ & China \\
\hline 29 & 7C-060 & China & 59 & $95-093$ & China \\
\hline 30 & 7C-090 & China & 60 & Golden delicious & USA \\
\hline
\end{tabular}

\section{Gradient screening and primer selection}

The annealing temperature is a key element of conventional PCR techniques. To ensure high repeatability and stability of SSR-PCR technology, it is essential to perform a gradient filter of annealing temperature for different primers. PCR was then performed according to the method of Yu et al. (2009). In order to ensure the quality and integrity of PCR products, primers with clear and highly repeated bands were selected based on 3 consecutive gradients, and the higher temperature was chosen for the appropriate selection.

\section{PCR amplification}

These primers were used to conduct PCR amplification. PCR amplification was carried out in a $20-\mu \mathrm{L}$ reaction system containing $2 \mu \mathrm{L} 30 \mathrm{ng} / \mu \mathrm{L}$ genomic DNA, $0.8 \mu \mathrm{L} 10$ $\mu \mathrm{M}$ of each primer, $0.1 \mu \mathrm{L} 5 \mathrm{U} / \mu \mathrm{L}$ Taq DNA polymerase, $2 \mu \mathrm{L} 10 \mathrm{X}$ buffer, $1.6 \mu \mathrm{L} 25 \mathrm{mM}$ $\mathrm{MgCl}_{2}$, and $1.2 \mu \mathrm{L} 2.5 \mathrm{mM}$ dNTPs. The amplification of the reaction was performed in an Eppendorf Authorized Thermal Cycler using the following temperature cycling parameters: initial denaturation for $5 \mathrm{~min}$ at $94^{\circ} \mathrm{C} ; 35$ cycles of denaturation at $94^{\circ} \mathrm{C}$ for $40 \mathrm{~s}$, corresponding annealing temperature for $40 \mathrm{~s}$, and extension at $72^{\circ} \mathrm{C}$ for $1 \mathrm{~min}$; and a final extension step at $72^{\circ} \mathrm{C}$ for $10 \mathrm{~min}$. PCR products were resolved on non-denaturing polyacrylamide gel electrophoresis to check the DNA banding patterns ( $\mathrm{Li}$ et al., 2012). In order to have reproducible, accurate, and clear banding patterns, all amplifications were repeated separately at 
least three times. The PCR products were detected on $0.8 \%$ agarose gels, then we used an $8 \%$ non-denaturing polyacrylamide gel electrophoresis instrument vertical plate and DYC-30 type electrophoresis tank to separate the bands at $200 \mathrm{~V}$ and $100 \mathrm{~mA}$ for $90 \mathrm{~min}$, and bands were visualized with silver staining.

\section{Detection of the SSR polymorphic bands and data statistics}

Only clear unambiguous SSR polymorphic bands in the photographic prints of gels were chosen and scored for cultivar identification (Yu et al., 2009). Cultivars having a specific band in the fingerprint generated from one primer pair could be separated singly, with cultivars sharing the same banding pattern being separated into the same subgroup. Based on these criteria, all apple cultivars were gradually separated from each other as more primers were employed, and a final apple manual CID was constructed following the strategy reported by Zhao et al. (2011).

\section{Test of utilization and workability of the CID in cultivar identification}

Three groups of apple cultivars were randomly chosen and used to verify the use and workability of the CID showing the separation of the 60 cultivars. The 3 groups of cultivars were marked $\mathrm{A}, \mathrm{B}$, and $\mathrm{C}$, and the corresponding primers used to separate each group were easily picked from the diagram. If the randomly selected cultivars could be distinguished accurately and quickly as anticipated, it would assure that the strategy developed and employed in this study is scientific, workable, and efficient. This approach is an ideal way to use molecular markers to identify not only fruit crop cultivars but also other crops. The PCR was performed as described above.

\section{RESULTS}

\section{Optimization of the SSR technology system}

To attain the exact primer annealing temperature information and polymorphic and stable bands, gradient screening and primer selection experiments were first carried out. The following SSR-PCR program was used: $94^{\circ} \mathrm{C}$ for $5 \mathrm{~min} ; 35$ cycles of denaturation at $94^{\circ} \mathrm{C}$ for $45 \mathrm{~s}$, gradient annealing temperature $\left(55\right.$ to $\left.60^{\circ} \mathrm{C}\right)$ for $40 \mathrm{~s}$, and $72^{\circ} \mathrm{C}$ for $1 \mathrm{~min}$; and $72^{\circ} \mathrm{C}$ for $10 \mathrm{~min}$. Annealing temperatures for each primer were screened based on the quality and reproducibility of banding patterns. The annealing temperature was the key factor influencing the stability of SSR-PCR. Once a positive primer that could produce clear, reproducible polymorphic bands was screened, it was used further in the identification of apple cultivars. The experiment results showed that 8 of 20 primer pairs may be used to identify apple cultivars. The fingerprints from the primers screened were polymorphic and stable, with the SSR-PCR product size ranging from 200 to $1000 \mathrm{bp}$, indicating the reliability of the optimized SSR-PCR systems and the fingerprints that were generated.

\section{Cultivar identification}

By the time the 8th primer pair was screened and used (Table 2), all the 60 apple 
cultivars could be successfully identified. An example of the SSR-PCR patterns in the study includes that obtained using primer Y5 (Figure 1), which was also the first primer used to amplify the 60 apple cultivars. The electrophoresis results showed that primer Y5 generated uniform, clear, and reproducible band patterns in 18 apple cultivars assigned with lane number codes $8,9,11,15,16,26,30,32,35,39,40,41,44,48,54,55,56$, and 58 (the lane numbers correspond to the cultivar names as shown in Table 1). This group of 18 apple cultivars was easily differentiated from the other 42 cultivars by the presence or absence of a distinct 530- or 350-bp band, causing all the 60 cultivars to be separated into 2 groups. The second primer (Y16) could further separate the 2 groups of cultivars earlier identified by the primer Y5 into smaller groups. After these, other primers were chosen to differentiate the several subgroups of apple cultivars. For example, the group of 16 cultivars $(1,7,10,12,13,17,19$, $22,27,28,42,43,46,50,52$, and 57) could be separated into 2 smaller groups and singly as 50 with band sizes of about 750, 400, and 360 bp by primer Y12 (Figure 2). Following this method systematically and utilizing the primers, we can differentiate all the 60 apple cultivars. All primer pairs were screened to differentiate the apple cultivars until full separation was achieved as shown in Figure 3.

Table 2. Eight primer pairs were chosen for further fingerprinting of the 60 apple genotypes.

\begin{tabular}{llc}
\hline Primer No. & Primer sequences $\left(5^{\prime} \rightarrow 3^{\prime}\right)$ & Tm $\left({ }^{\circ} \mathrm{C}\right)$ \\
\hline Y5 & ATATCTTCTCCCTGCTGCAAAG & 54.3 \\
Y9 & CATGGAATGAGTGGAGAAACAA & 55.2 \\
& ATGCGATCTCGAAGCATATACA & 53.9 \\
Y10 & ACCTTAGCACCTGTCAAGGGTA & 55.6 \\
Y12 & TGCAAACAATGGAAGTCTTGTC & 5 \\
& GGCCGCTAGATACAAGAAGAGA & 53.6 \\
Y15 & AACAACGACGTCCTCCACTATT & 52.8 \\
Y16 & AACAACCCATCACCTCAACTCT & 54.0 \\
Y18 & GCTATAGCTATGCTGCTCATGC & 54.0 \\
Y22 & CTGATCGAGATCATCACAAACG & 53.1 \\
& GCACCTCGGCAATGATACC & 5 \\
\hline
\end{tabular}

\section{Test of the use and workability of the CID}

This study aimed not only to generate a diagram like the work of cluster analysis for some cultivars, but also to generate a referable apple CID that could be used in the practical identification of some of these cultivars in the future in the nursery industry as well as in cultivar protection. Based on these two aims, it was important to verify the use, workability, and efficiency of the CID.

To undertake this, 3 groups of cultivars composed of 5,21,33, and 47; 31 and 36; and 16 and 48, which came from the inter-groups in the CID, were randomly chosen and used for the verification exercise. From the location of these cultivars in the CID, it was easy to determine that primers Y9, Y15, and Y22; Y10; and Y18 would be suitable to separate these 3 groups of cultivars. The PCR results of these 3 groups of cultivars using the corresponding primers were separated accordingly (Figure 4). 


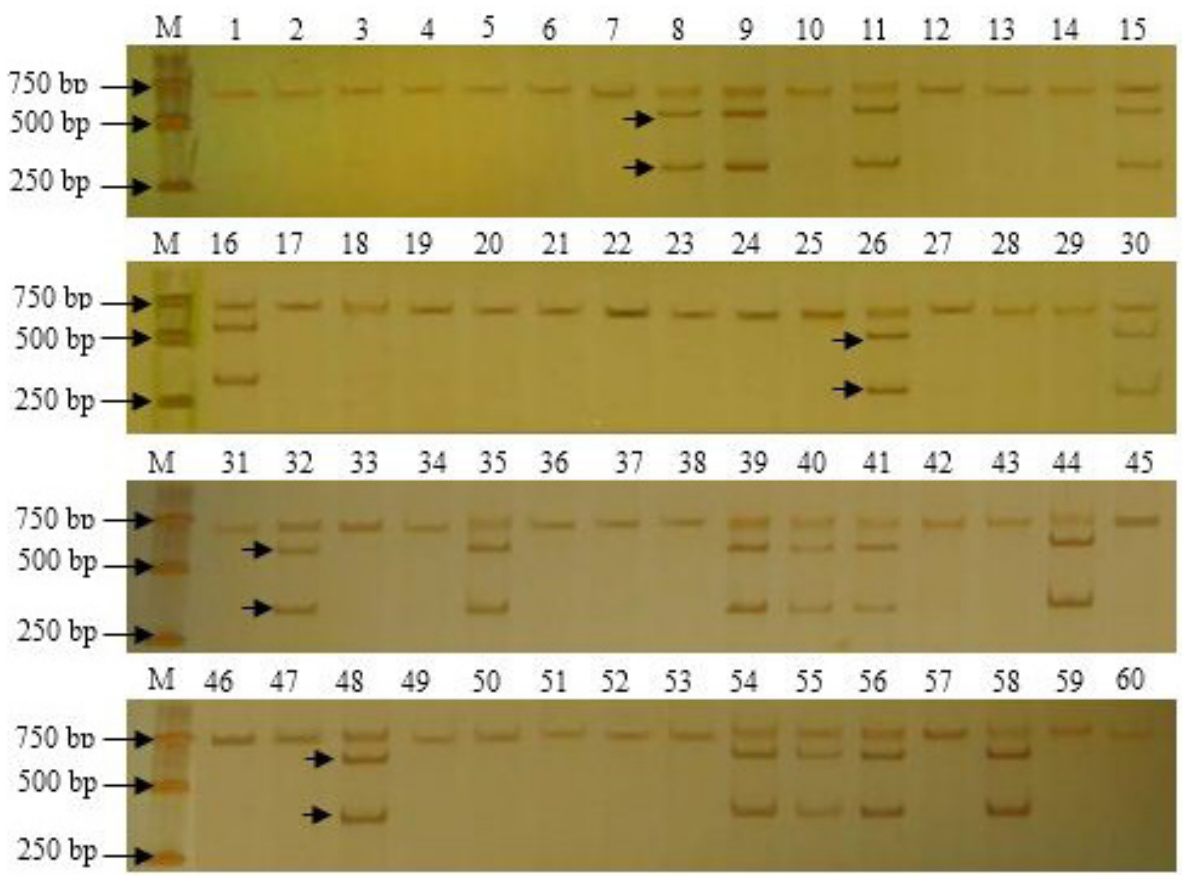

Figure 1. DNA banding patterns of 60 apple cultivars amplified by primer Y5. Small horizontal arrows indicate the specific bands. The lane numbers correspond to the cultivar code in Table 1. Lane $M=$ DL2000 plus marker.

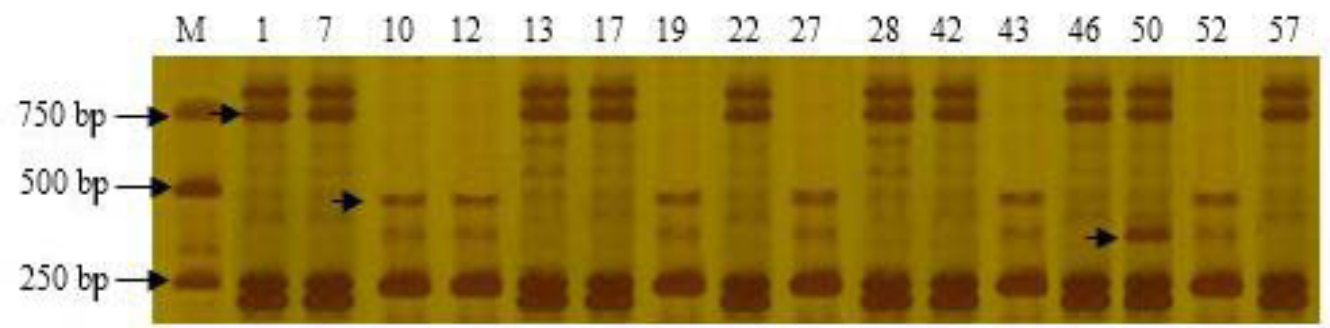

Figure 2. DNA banding patterns of 16 apple cultivars in group from the separation of primer Y12. Lane $M=$ DL2000 plus marker. The lane numbers correspond to the cultivar code in Table 1. The bands pointed with larger arrows were those employed to separate the cultivars.

The 1st group can be validated with 3 primers, Y9, Y15, and Y22. The PCR results showed that 4 apple cultivars could first be separated into 2 groups by primer Y 9 with the band of about 560 or $440 \mathrm{bp}$. One group made up of 21 and 47 can be further separeted by the 350$\mathrm{bp}$ band from primer Y15. The other group of 5 and 33 could then be divided by the 250-bp band of primer Y22 (Figure 4A). The 2nd group could be separated with a specific band about $410 \mathrm{bp}$ from primer Y10 as in the CID (Figure 4B). The 3rd group was divided by primer Y18 with the band of about 450 bp (Figure 4C). Using this procedure, all the 3 groups were successfully identified with the suitable combination of primers. This test therefore proves the availability, workability, and efficiency of this method in apple cultivar identification. 


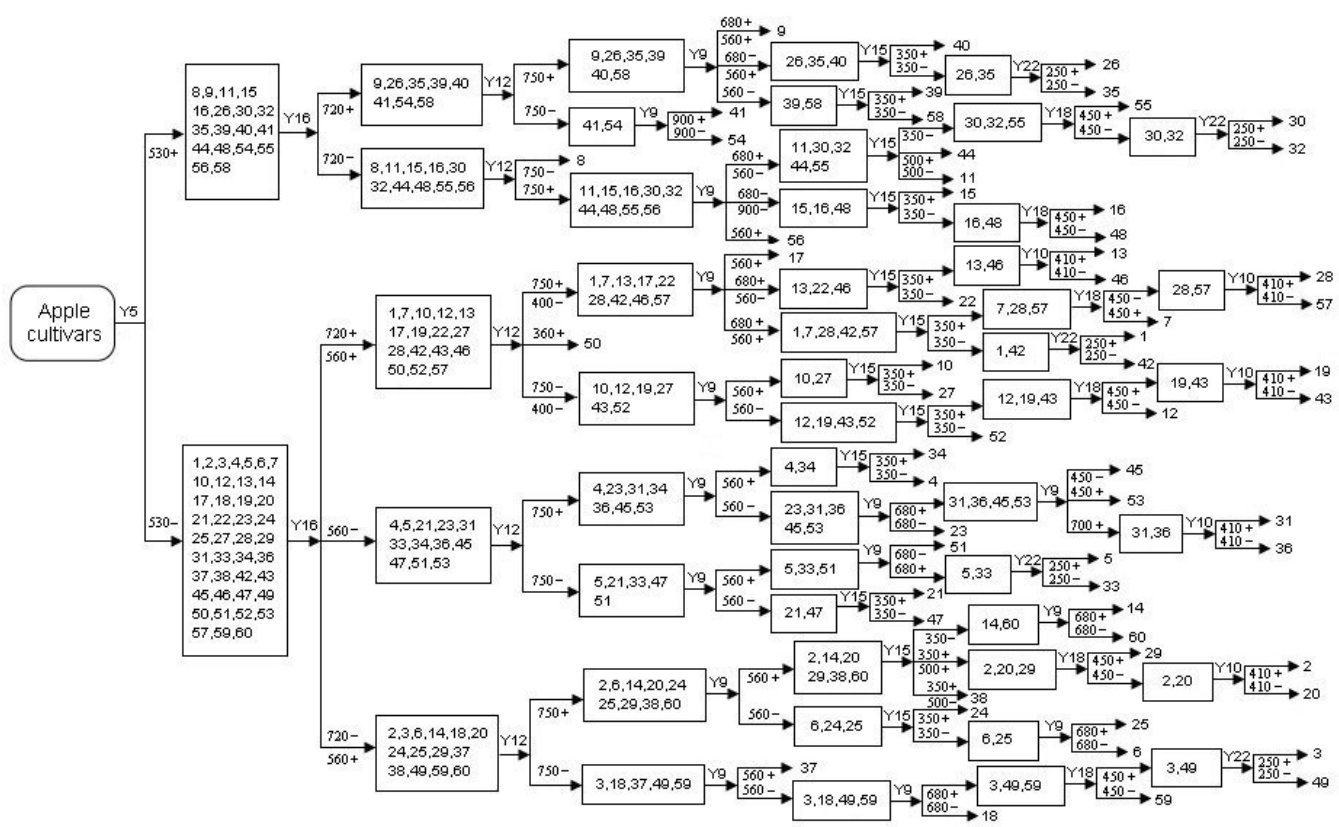

Figure 3. Results of identification of apple cultivars by 8 primer pairs and detailed fingerprints. All the numbers marked in this chart indicate different sized fingerprints, and the unit is bp; $+=$ presence of bands; - = absence of bands.
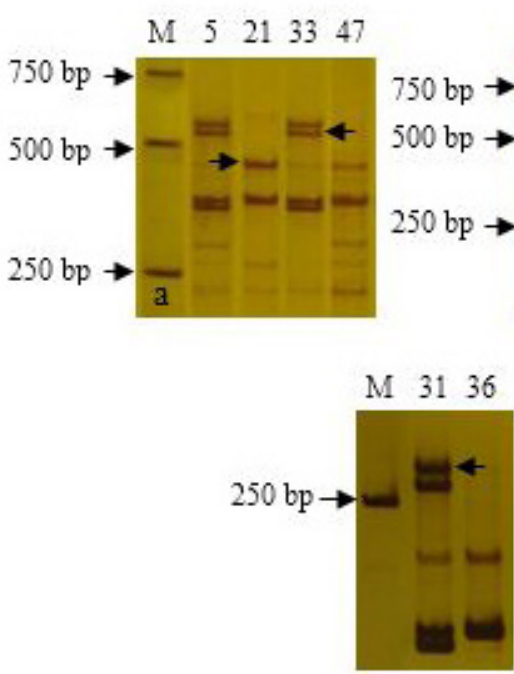

B
M 2147

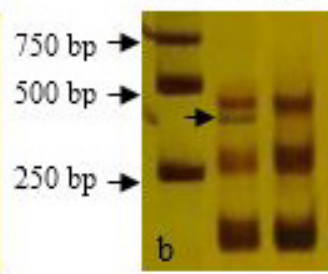

A

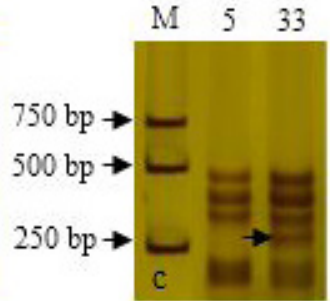

M 1648

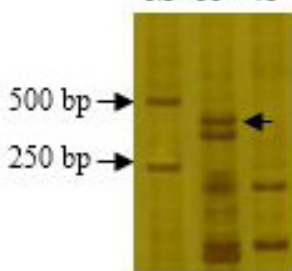

$\mathrm{C}$

Figure 4. Verification result of three groups cultivars selected randomly by the corresponding primers. Lane $M=$ DL2000 plus marker; The lane numbers correspond to the cultivar code in Table 1; A. fingerprint obtained with the three primers used to separate the first group of cultivars selected. a, b, and c obtained with the primer Y9, Y15, and Y22 respectively; B. fingerprint obtained with the primer Y10 used to separate the ' 31 , ' 36 '; C. DNA fingerprint obtained with the primer Y18 used to separate the group '16' and '48'. 


\section{DISCUSSION}

In China, there is a great number of apple cultivars at present. In addition to cultural varieties, China has other apple germplasm resources, including a large number of wild species, half-wild species, and cultivars. These germplasm resources and conservation programs need exact names. However, because of the frequent exchanges of apple cultivars in the world, there are synonyms for one apple cultivar or one name for different apple cultivars. The name confusion directly affect future production of desired cultivars. Therefore, it is necessary to find an exact method that can distinguish apple cultivars accurately and easily in practice. Although thousands of papers on cultivar identification have been published since several generations of DNA markers are developed, it is not easy to find the most effective and efficient way that can service the identification of the cultivars. Usually, the situation is not anticipated and satisfied. Its key question is whether DNA markers can distinguish plant varieties efficiently and easily in practice. Molecular markers, including RAPD (Roche et al., 1997; Palombi and Damiano, 2002; Stark-Urnau, 2002; Silfverberg-Dilworth et al., 2006), SSR (Palombi and Damiano, 2002), and AFLP (Roche et al., 1997), have been used in the genetic analysis of variety relationships and organisms. However, the phylogenetic trees derived from cluster analysis cannot clarify which information can be used to identify plant samples. In addition, fingerprinting cannot present all the fingerprints from many cultivars together for identification. A very significant reason is that no analysis could connect the information of DNA fingerprints with cultivars in an easy, clear, and readable way.

CID based on DNA markers was developed and succeeded in identifying a lot of fruit crops (Wang et al., 2011; Leng et al., 2012). This method can facilitate the use of the DNA markers on plant cultivar identification. If polymorphic bands from each primer are used to screen and gradually distinguish the individual samples, the identification results will be charted informatively and clearly, and it is not necessary to observe the morphology traits of the fruit tree. From the agronomic point of view, the CID can be put into use in many fields, including fruit exportation, early seed identification, and seed purity assessment. The new approach developed to use DNA markers to distinguish cultivars is both efficient and practical, yielding low cost, quick work, and clear aim, among other benefits. There are a lot of DNA markers to suit to CID. Among them, SSR markers have become valuable molecular tools for genetic fingerprinting because of their abundance, high degree of polymorphism, cross transference, co-dominant nature and suitability for automation. An additional advantage is that SSR markers have an extensive source.

This is the first report on using CID to identify apple cultivars. In this study, we focused on the use of DNA fingerprints based on SSRs to identify apple cultivars and to test the transferability of the CID. The results showed that only 8 pairs of SSR primers were needed to distinguish all the 60 apple cultivars. The new CID strategy in this study could clearly make the most out of the polymorphic SSR-PCR bands for efficient identification of apple cultivars, which overcomes the drawbacks of the cluster analyses previously employed in plant identification. The informative CID (Figure 3) of apple cultivars is the key result that can tell us which primer or primers can be used to separate target apple cultivars. Basically, any 2 cultivars can be identified with an SSR primer. In practice, if new apple cultivars are released, the set of 8 SSR primer pairs can be used in the DNA analysis of the new cultivars, and the PCR banding patterns can indicate the position of the new cultivars in the CID. If the 8 SSR primer pairs 
cannot differentiate the new cultivars, some new primers can be screened and used to separate these cultivars and position them on the CID, leading to the generation of a larger CID. It seems that it is not needed a great effort to separate one or several new cultivars. Finally, the CID generated can work just like the periodic table of elements in chemistry, applying in practice (Leng et al., 2012). In order to verify the reliability of this theory, experimental verification is necessary. The verification of the function and accuracy of the CID as anticipated can confirm the practical importance of this cultivar identification method. For any 2 or a few cultivars, if we choose corresponding primers, we can distinguish them from each other by specific SSR-PCR bands. We believe that this separation of cultivars and the new strategy will definitely be significant in the apple industry in China.

The use of such a CID in plant cultivar identification has some advantages. It only needs few corresponding primers to make all cultivars efficiently separated. The related experiments are easy to operate. Whenever the referable information that the CID yields, it can be used. Therefore, it is not just a simple theoretical model, but a truly useful model that makes DNA markers more applicable for plant variety identification in practice. Moreover, the polymorphic bands generated may be developed into special molecular markers for cultivar identification in the future. The CID associated with SSR markers make it possibile to utilize DNA markers to distinguish the cultivars in any species having a highly heterozygous genome, without the need for a genetic linkage map or any DNA sequence information (Lin et al., 2011). We anticipate that this a method can be used to draw CIDs for each organism, whether plant, animal, or any other creature and provide useful information to separate cultivars successfully.

In conclusion, this method conveniently produces reliable results. The experiment results demonstrated that a standard set of primers can be used to distinguish many Malus domestica cultivars. Meanwhile, another step for the identification is polymorphic and specific primer screening. Once SSR markers are developed from the genome sequence, cDNA library and EST databases, the CIDs will be established and used for preventing from identifying errors, evaluating new potential accessions, and ensuring maximum diversity of core collections following the method as reported (Wang et al., 2011; Lin et al., 2011; Leng et al., 2012).

\section{ACKNOWLEDGMENTS}

Research supported by the China Agriculture Research System Foundation (\#CARS28-01-07) and the Shandong Provincial Improved Variety Engineering System Foundation (\#620902).

\section{REFERENCES}

Bousquet J, Simon L and Lalonde M (1990). DNA amplification from vegetative and sexual tissues of tree using polymerase chain reaction. Can. J. For. Res. 20: 254-257.

Brooks HJ and Vest G (1985). Public programs on genetics and breeding of horticultural crops in the United States. HortScience 20: 826-830.

Cheng ZP and Huang HW (2009). SSR fingerprinting Chinese peach cultivars and landraces (Prunus persica) and analysis of their genetic relationships. Sci. Hortic. 120: 188-193.

Donini P, Stephenson P, Bryan GJ and Koebner RMD (1998). The potential of microsatellites for high throughput genetic diversity assessment in wheat and barley. Genet. Resour. Crop Evol. 45: 415-421.

Elidemir AY and Uzun I (2009). Assessment of genetic diversity of some important grape cultivars, rootstocks, and wild 
grapes in Turkey using RAPD markers. Acta Hortic. 827: 275-278.

Ferreira M and Grattapaglia D (1995). Introdução ao Uso de Marcadores RAPD e RFLP em Análise Genética. Embrapa Cenargen, Brasília, 1-220.

Hummer KE and Janick J (2009). Rosaceae: Taxonomy, Economic Importance, Genomics. In: Genetics and Genomics of Rosaceae (Folta K and Gardiner S, eds.). Springer, New York, 1-17.

Janick J, Cummins JN, Brown SK and Hemmat M (1996). Apples. In: Fruit Breeding, Tree and Tropical Fruits (Janick J and Moore JN, eds.). John Wiley and Sons, New York, 7-77.

Leng XP, Li HR, Zhong GY and Song CN (2012). Employment of a new strategy for identification of loose-skin mandarin (Citrus reticulata Blanco) cultivars using RAPD markers. Rom. Biotechnol. Lett. 17: 7073-7083.

Li HR, Wang K, Sun X and Zhang CH (2012). Development of EST-derived SSR markers and their applications in apple genetic diversity analysis. Jiangsu J. Agr. Sci. 28: 158-165.

Lin J, Wang XC, Chang YH and Fang JG (2011). Development of a novel and efficient strategy for practical identification of Pyrus spp (Rosaceae) cultivars using RAPD fingerprints. Genet. Mol. Res. 10: 932-942.

Melgarejo P, Martínez JJ, Hernández F and Martínez R (2009). Cultivar identification using 18S-28S rDNA intergenic spacer-RFLP in pomegranate (Punica granatum L.). Sci. Hortic. 120: 500-503.

Murray MG and Thompson WF (1980). Rapid isolation of high molecular weight plant DNA. Nucleic Acids Res. 8: 43214325.

Nybom H and Schaal BA (1990). DNA "fingerprints" applied to paternity analysis in apples (Malus x domestica). Theor. Appl. Genet. 79: 763-768.

Palombi MA and Damiano C (2002). Comparison between RAPD and SSR molecular markers in detecting genetic variation in kiwifruit (Actinidia deliciosa A. Chev). Plant Cell Rep. 20: 1061-1066.

Papa R, Troggio M, Ajmone-Marsan P and Nonnis MF (2005). An improved protocol for the production of AFLP markers in complex genomes by means of capillary electrophoresis. J. Anim. Breed Genet. 122: 62-68.

Papp N, Szilvássy B, Abrankó L and Szabó T (2010). Main quality attributes and antioxidants in Hungarian sour cherries: identification of genotypes with enhanced functional properties. Int. J. Food Sci. Technol. 45: 395-402.

Powell W, Morgante M, Andre C and Hanafey M (1996). The comparison of RFLP, RAPD, AFLP and SSR (microsatellite) markers for germplasm analysis. Mol. Breed. 2: 225-238.

Ramsay L, Macaulay M, degli Ivanissevich S and MacLean K (2000). A simple sequence repeat-based linkage map of barley. Genetics 156: 1997-2005.

Roche P, Alston FH, Maliepaard C and Evans KM (1997). RFLP and RAPD markers linked to the rosy leaf curling aphid resistance gene (Sdl) in apple. Theor. Appl. Genet. 94: 528-533.

Röder MS, Korzun V, Wendehake K and Plaschke J (1998). A microsatellite map of wheat. Genetics 149: 2007-2023.

Silfverberg-Dilworth E, Matasci CL, Van de Weg WE and Van Kaauwen MPW (2006). Microsatellite markers spanning the apple (Malus x domestica Borkh.) genome. Tree Genet. Gen. 2: 202-224.

Smith JSC, Chin ECL, Shu H and Smith OS (1997). An evaluation of the utility of SSR loci as molecular markers in maize (Zea mays L.): comparisons with data from RFLPs and pedigree. Theor. Appl. Genet. 95: 163-173.

Stark-Urnau M (2002). Use of RAPD-markers in Malus x domestica (apple) and Pyrus communis (pear) for cultivar identification - Part I: Malus x domestica (apple). Erwerbsobstbau 44: 139-144.

Temnykh S, DeClerck G, Lukashova A and Lipovich L (2001). Computational and experimental analysis of microsatellites in rice (Oryza sativa L.): frequency, length variation, transposon associations, and genetic marker potential. Genome Res. 11: 1441-1452.

Varshney RK, Graner A and Sorrells ME (2005). Genic microsatellite markers in plants: features and applications. Trends Biotechnol. 23: 48-55.

Wang YJ, Li XY, Han J and Fang WM (2011). Analysis of genetic relationships and identification of 54 flowering-mei cultivars using EST-SSR markers developed from apricot and fruiting-mei. Sci. Hortic. Amsterdam 132: 12-17.

Watillon B, Druart P, Du Jardin P and Kettmann R (1991). Use of random cDNA probes to detect restriction fragment length polymorphisms among apple clones. Sci. Hortic. Amsterdam 46: 235-243.

Williams JG, Kubelik AR, Livak KJ, Rafalski JA, et al. (1990). DNA polymorphisms amplified by arbitrary primers are useful as genetic markers. Nucleic Acids Res. 18: 6531-6535.

Wrigley CW, Batey IL, Campbell WP and Skerritt JH (1987). Complementing traditional methods of identifying cereal varieties with novel procedures. Seed Sci. Technol. 15: 679-688.

Yu HP, Fang JG, Zhang MY, Yang G, et al. (2009). Study on application of RAPD marker in cultivar identification of seven fruit crops. Acta Agr. Jiangxi 21: 5-9.

Zhao MZ, Zhang YP, Wu WM, Wang C, et al. (2011). A new strategy for complete identification of 69 grapevine cultivars using random amplified polymorphic DNA (RAPD) markers. Afr. J. Plant Sci. 5: 273-280. 